\title{
Гацаева P.C-А.
}

\section{Исследование одной функциональной, краевой задачи для дифференциального уравнения первого порядка}

Чеченский государственный университет (Россия, Грозный)

doi: 10.18411/lj-05-2019-04

idsp: ljournal-05-2019-04

\section{Аннотация}

В статье рассматривается специальная краевая задача. Доказывается теорема существования и единственности решения. Результаты находят применение при исследовании экстремальных свойств решений.

Ключевые слова: Специальная краевая задача; теорема существования; экстремальные свойства.

\section{Abstract}

The article deals with a special boundary value problem. The theorem of existence and uniqueness of the solution is proved. The results are used in the study of extreme properties of solutions.

Keywords: special boundary value problem; existence theorem; extreme properties.

Будем изучать дифференциальное уравнение вида

$$
\begin{aligned}
y^{\prime}+P(x) y & =f(x, y) \\
y^{\prime}\left(x_{0}\right) & =d
\end{aligned}
$$

где $x_{0} \in[a, b], \mathrm{P}(\mathrm{x}), f(x, y)$ непрерывные функции по всем переменным, причем $P\left(x_{0}\right) \neq 0, d-$ некоторое число. Если функция $y(x)$ есть решение уравнения (1), удовлетворяющее условию (2), то из (2) вытекает справедливость равенства

$$
y\left(x_{0}\right)=\left(P\left(x_{0}\right)\right)^{-1}\left[f\left(x_{0}, y\left(x_{0}\right)\right)-d\right]
$$

И наоборот, если функция $y(x)$ есть решение уравнения (1), удовлетворяющее условию (3), то имеет место и равенство (2). Следовательно ,задачи (1),(2) и (1),(3) эквивалентны, поэтому, чтобы выявить определенные свойства решения задачи(1),(2), будем изучать задачу (1),(3), которая относится к категории задач с нелинейными функциональными краевыми условиями

является частным случаем функциональной краевой задачи из [1].

Как будет показано ниже, задача (1),(3) имеет практическое приложение. Для доказательства теоремы существования и единственности решения этой задачи будет использован новый прием, основанный на сходимости одного , специальным образом построенного итерационного процесса, идея которого со значительной пользой применяется и при исследовании различных многоточечных и функциональных краевых задач.

Теорема. Пусть $P(x)$ функция непрерывна на $[a, b]$ и $P\left(x_{0}\right) \neq 0, x_{0} \subset[a, b]$; функция $f(x, y)$ непрерывна по совокупности переменных в замкнутой области D. Если выполнены неравенства

$$
|f(x, \bar{y})-f(x, \overline{\bar{y}})| \leq L_{1}(x)|\bar{y}-\overline{\bar{y}}|
$$

где $L_{1}(x)_{\text {- непрерывная функция на }}[a, b]$, для любых значений $\bar{y}, \overline{\bar{y}}$ переменного 


$$
M_{1}=|\alpha| L_{1}\left(x_{0}\right) l<1
$$

где

$$
\alpha=P^{-1}\left(x_{0}\right), \quad l=\max _{x \in[a, b]} \exp _{(}\left(\int_{a}^{x} L_{1}(t) d t-\int_{x_{0}}^{x} P(t) d t\right) ; \quad \frac{N_{1}}{1-M_{1}} \leq r,
$$

$N_{1}=\max _{x \in[a, b]}\left|\alpha\left[f\left(x_{0}, 0\right)-d\right]+\int_{x_{0}}^{x} f(t, 0) \exp \left(\int_{x_{0}}^{t} P(s) d s\right)\right|$.

Тогда задача (1),(2) имеет единственное непрерывное решение.

Доказательство. Возьмем функцию $y_{0}(x)=0$. За функцию $y_{1}(x)$ выберем решение интегрального уравнения

$$
y_{1}(x)=\alpha\left[f\left(x_{0}, 0\right)-d\right] \exp \left(-\int_{x_{0}}^{x} P(s) d s\right)+\int_{x_{0}}^{x} f\left(t, y_{1}(t)\right) \exp \left(\int_{x}^{t} P(s) d s\right) d t .\left(5_{1}\right)
$$

В качестве функции $y_{2}(x)$ возьмем решение интегрального уравнения

$$
\left.y_{2}(x)=\alpha\left[f\left(x_{0}, y_{1}\left(x_{0}\right)\right)-d\right] \exp \left(-\int_{x_{0}}^{x} P(s) d s\right)+\int_{x_{0}}^{x} f\left(t, y_{1}(t)\right) \exp \left(\int_{x}^{t} P(s) d s\right) d t \quad, \quad 5_{2}\right)
$$

и т.д. строить последовательность функции $y_{1}(x), y_{2}(x), \ldots$

$$
\begin{gathered}
y_{n}(x)=\alpha\left[f\left(x_{0}, y_{n-1}\left(x_{0}\right)\right)-d\right] \exp \left(-\int_{x_{0}}^{x} P(s) d s\right)+\int_{x_{0}}^{x} f\left(t, y_{n}(t)\right) \exp \left(\int_{x}^{t} P(s) d s\right) d t, \quad n=1,2, \ldots \\
y_{1}(x) \exp \left(\int_{x_{0}}^{x} P(s) d s\right)=N+\int_{x_{0}}^{x} f\left(t, y_{1}(t)\right) \exp \left(\int_{x}^{t} P(s) d s\right) d t \\
\text { Из }\left(5_{1}\right) \text { имеем } \\
\text { где } N=\alpha\left[f\left(x_{0}, 0\right)-d\right] . \text { Далее } \\
y_{1}(x) \exp \left(\int_{x_{0}}^{x} P(s) d s\right)=N+\int_{x_{0}}^{x} f(t, 0) \exp \left(\int_{x}^{t} P(s) d s\right) d t+\int_{x_{0}}^{x}\left[f\left(t, y_{1}(t)\right)-f(t, 0)\right] \exp \left(\int_{x_{0}}^{t} P(s) d s\right)
\end{gathered}
$$

Поэтому , ввиду (4) , получим

$$
\begin{gathered}
\left.\left|y_{1}(x)\left(\exp \int_{x_{0}}^{x} P(s) d s\right)\right|=\mid N+\int_{x_{0}}^{x} f(t, 0)\right) \exp \left(\int_{x}^{1} P(s) d(s)\right) d t \mid+ \\
\int_{a}^{x} L_{1}(t)\left|y_{1}(t)\right| \exp \left(\int_{x_{0}}^{1} P(s) d s\right) d t
\end{gathered}
$$

Следовательно ,справедлива оценка [1.2]

$$
\begin{gathered}
\left|y_{1}(x)\right|\left(\exp \int_{x_{0}}^{x} P(s) d s\right) \leq \\
\left.N_{1}=\mid N+\int_{a} \exp \left(\int_{a}^{x} L_{1}(t) d t\right), 0\right)\left|\exp \left(\int_{x_{0}}^{t} P(s) d s\right) d t\right| .
\end{gathered}
$$


Из (7) имеем

$$
\left|y_{1}(x)-y_{0}(x)\right| \leq N_{1} \exp \left(\int_{a}^{x} L_{1}(t) d t-\int_{x_{0}}^{x} P(t) d t\right) \leq N_{1} l
$$

Из $\left(5_{1}\right)$ и $\left(5_{2}\right)$ находим

$\left[y_{2}(x)-y_{1}(x)\right] \exp \left(\int_{x_{0}}^{x} P(s) d s\right)=\alpha\left[f\left(x_{0}, y_{1}\left(x_{0}\right)\right)-f\left(x_{0}, 0\right)\right]+$

$+\int_{x_{0}}^{x}\left[f\left(t, y_{2}(t)\right)-f\left(t, y_{1}(t)\right)\right] \exp \left(\int_{x_{0}}^{t} P(s) d s\right) d t$.

Следовательно,

$$
\begin{aligned}
& \left|y_{2}(x)-y_{1}(x)\right| \exp \left(\int_{x_{0}}^{x} P(s) d s\right) \leq|\alpha| L_{1}\left(x_{0}\right)\left|y_{1}\left(x_{0}\right)\right|+ \\
& \int_{a}^{x} L_{1}(t)\left|y_{2}(t)-y_{1}(t)\right| \exp \left(\int_{x_{0}}^{t} P(s) d s\right) d t \\
& \text { или [2] (см. ( } \left.\left.6_{1}\right)\right) \\
& \left|y_{2}(x)-y_{1}(x)\right| \exp \left(\int_{x_{0}}^{x} P(s) d s\right) \leq \\
& |\alpha| L_{1}\left(x_{0}\right)\left|y_{1}\left(x_{0}\right)\right|\left(\int_{a}^{x} L_{1}(t) d t\right) \leq \\
& |\alpha| L_{1}\left(x_{0}\right) N_{1} l \exp \left(\int_{x_{0}}^{t} P(s) d s\right) d t . \\
& \text { Отсюда }\left|y_{2}(x)-y_{1}(x)\right| \leq|\alpha| L_{1}\left(x_{0}\right) N_{1} l \exp \left(\int_{a}^{x} L_{1}(t) d t-\int_{x_{0}}^{x} P(t) d t\right) \leq|\alpha| L_{1}\left(x_{0}\right) N_{1} l M_{1} . \\
& \text { АналОгично }
\end{aligned}
$$

Аналогично

$$
\begin{aligned}
& \left|y_{3}(x)-y_{2}(x)\right| \exp \left(\int_{x_{0}}^{x} P(s) d s\right) \leq \\
& |\alpha| L_{1}\left(x_{0}\right)\left|y_{2}\left(x_{0}\right)-y_{1}\left(x_{0}\right)\right|+\int_{a}^{x} L_{1}(t)\left|y_{3}(t)-y_{2}(t)\right| \exp \left(\int_{x_{0}}^{t} P(s) d s\right) d t, \\
& \left|y_{3}(x)-y_{2}(x)\right| \exp \left(\int_{x_{0}}^{x} P(s) d s\right) \leq|\alpha| L_{1}\left(x_{0}\right)\left|y_{2}\left(x_{0}\right)-y_{1}\left(x_{0}\right)\right| \exp \left(\int_{a}^{x} L_{1}(t) d t\right) \leq \\
& \leq|\alpha| L_{1}(x)|\alpha| L_{1}\left(x_{0}\right) N_{1} l^{2} \exp \left(\int_{a}^{x} L_{1}(t) d t\right),
\end{aligned}
$$

следовательно, $\quad\left|y_{3}(x)-y_{3}(x)\right| \leq|\alpha|^{2} L_{1}^{2}\left(x_{0}\right) N_{1} l M_{1}^{2}$,

и т.д. для любого $\mathrm{n}$ имеет оценку

$$
\left|y_{3}(x)-y_{3}(x)\right| \leq N_{1}|\alpha|^{n-1} L_{1}^{n-1}\left(x_{0}\right) l^{n}=N_{1} l M_{1}^{n-1},
$$

Если предположить , что выполняется неравенство (6) то, как нетрудно проверить , не одна из функций $y_{1}(x), y_{2}(x), \ldots$ не выходит из области $D_{i}$ т.е.

$\left|y_{n}(x)\right| \leq r$ для любого $n$.

Пусть $M_{1}<1$. Тогда функциональный ряд

$$
\sum_{n=1}^{\infty}\left[y_{n}(x)-y_{n-1}(x)\right]
$$


мажорируется сходящимся числовым рядом $\sum_{n=1}^{\infty} N_{1} l M_{1}^{n-1}$

и , следовательно, равномерно сходится на сегменте [a,b]. обозначим сумму ряда (8) через $y(x)$. Очевидно, что

$$
\lim _{n \rightarrow \infty} y_{n}(x)=y(x)
$$

и $y(x)$ есть непрерывная функция. Переходим к пределу в $\left(5_{n}\right)$ при $n \rightarrow \infty$, в силу (9) имеем

$$
y(x)=\alpha\left[f\left(x_{0}, y\left(x_{0}\right)\right)-d\right] \exp \left(-\int_{x_{0}}^{x} P(s) d s\right)+\int_{x_{0}}^{x} f(t, y(t)) \exp \left(\int_{x}^{t} P(s) d s\right) d t
$$

Дифференцируя обе части (10) по $x$, получим

$$
\begin{aligned}
& y^{\prime}(x)=-P(x) \alpha\left[f\left(x_{0}, y\left(x_{0}\right)\right)-d\right] \exp \left(-\int_{x_{0}}^{x} P(s) d s\right)+ \\
& f(x, y(x))+P(x) \int_{x_{0}}^{x} f(t, y(t)) \exp \left(\int_{x}^{t} P(s) d s\right) d t= \\
& =-P(x) \alpha\left[f\left(x_{0}, y\left(x_{0}\right)\right)-d\right] \exp \left(-\int_{x_{0}}^{x} P(s) d s\right)+f(x, y(x))-P(x) y(x)+ \\
& +P(x) \alpha\left[f\left(x_{0}, y\left(x_{0}\right)\right)-d\right] \exp \left(-\int_{x_{0}}^{x} P(s) d s\right)=P(x) y(x)+f(x, y(x)),
\end{aligned}
$$

т.е. функция $y(x)$ является решением дифференциального уравнения (10).

при $x=x_{0}$ из (10) следует $y(x)=\alpha\left[f\left(x_{0}, y\left(x_{0}\right)\right)-d\right]$

или $P\left(x_{0}\right) y\left(x_{0}\right)=f\left(x_{0}, y\left(x_{0}\right)\right)-d$,

Значит $y^{\prime}\left(x_{0}\right)=d$.

Таким образом, при перечисленных предположениях задача (1), (2) имеет хотя бы одно решение. Этих предположений вполне достаточно, чтобы решение было единственным.

Действительно пусть задача (1), (2) имеет два решение $y(x), z(x)$. Тогда имеет место тождество (см.(10)).

Отсюда

$$
\begin{aligned}
& y(x)-z(x)=\alpha\left[f\left(x_{0}, y\left(x_{0}\right)\right)-f\left(x_{0}, z\left(x_{0}\right)\right)\right] \exp \left(-\int_{x_{0}}^{x} P(s) d s\right)+ \\
& +\int_{x_{0}}^{x}[f(t, y(t))-f(t, z(t))] \exp \left(\int_{x_{0}}^{t} P(s) d s\right) d t .
\end{aligned}
$$

или по лемме Гронуолла-Беллмана [1]

$$
\begin{aligned}
& |y(x)-z(x)| \exp \left(\int_{x_{0}}^{x} P(s) d s\right) \leq|\alpha| L_{1}\left(x_{0}\right)\left|y\left(x_{0}\right)-z\left(x_{0}\right)\right|+ \\
& \int_{a}^{x} L_{1}(t)|y(t)-z(t)| \exp \left(\int_{x_{0}}^{t} P(s) d s\right) d t
\end{aligned}
$$

Следовательно

$$
|y(x)-z(x)| \exp \left(\int_{x_{0}}^{x} P(s) d s\right) \leq|\alpha| L_{1}\left(x_{0}\right)\left|y\left(x_{0}\right)-z\left(x_{0}\right)\right| \exp \left(\int_{a}^{x} L_{1}(t) d t\right),
$$

$$
|y(x)-z(x)| \leq\left|y\left(x_{0}\right)-z\left(x_{0}\right)\right| M_{1},
$$




$$
\begin{gathered}
\max _{x \in[a, b]}|y(x)-z(x)| \leq \max _{x \in[a, b]}|y(x)-z(x)| M_{1}, \\
M_{1} \geq 1,
\end{gathered}
$$

чем быть не может в силу неравенства (5).

Итак, теорема доказана.

$$
\text { *** }
$$

1. Исраилов С.В., Юшаев С.С. Многоточечные и функциональные краевые задачи дм обыкновенных дифференциальных уравнений. Нальчик, Изд-во «Эльфа», 2004. С445

2. Сансоне Дж. Обыкновенные дифференциальные уравнения. Т.1.2., Москва, ИЛ,1954 